\title{
SEMI-AUTOMATIC MULTI-CAMERA VIDEO ANNOTATION TOOL FOR OBJECT TRACKING AND OPTICAL FLOW BENCHMARK CREATION
}

\author{
Vladimir V. Devyatkov and Igor I. Lychkov \\ Bauman Moscow State Technical University, Vtoraya Baumanskaya 5, Moscow, Russia
}

\begin{abstract}
This paper addresses a problem of video annotation for object tracking evaluation. Contemporary object tracking methods relies heavily on optical flow calculation techniques. State of the art optical flow calculation methods are not enough to provide robust and accurate tracking under object occlusion and shading conditions. Thus, comprehensive visual object tracking datasets are required for deep analysis, benchmarking and improvement of object tracking methods. In most cases publicly available real video datasets does not include ground truth for optical flow evaluation, so new datasets might be in demand. Public video annotation tools for object tracking dataset labeling were reviewed. Lack of open source video annotation tools with functions of automatic optical flow markers and object bounding box visual tracking was noticed. In this work we developed a new open video annotation tool that is suitable for object bounding box labeling as well as optical flow annotation in multicamera video recordings. This tool makes it possible to specify the feature point and/or moving object position on the first video frame and track its position automatically in the subsequent video frames. This tool supports manual adjustment of the tracked position if needed. Multi-layer modular architecture of the annotation tool simplifies its testing, maintenance and further extension by the community.
\end{abstract}

\section{KEYWORDS}

Moving Object Tracking, Multi-Camera Video, Optical Flow, Video Annotation Tool

\section{INTRODUCTION}

Visual object tracking is a challenging task related to various problem domains including self-driving cars, security, robotics, etc. Broad scientific research in visual object tracking started in 1980s and is still in progress (Anishchenko, 2018; Deviatkov and Lychkov, 2017; Lychkov et al, 2018; Morozov et al, 2015; Taranyan et al, 2018) because real videos include many issues that are difficult to deal with, e. g. moving object occlusion, drastic illumination changes, video noise and blur. Main approaches to visual object tracking are blob-tracking (Wu et al, 2018), optical flow based tracking (Du et al, 2018), neural network tracking (Price et al, 2018), contour-based tracking (Allili and Ziou, 2006), 3D model based tracking (Yu et al, 2018), etc. Neural networks outperforms other approaches in terms of accuracy but they require special hardware for real-time operation. Object tracking methods implemented in real-time embedded systems are mostly based on optical flow techniques which are less computationally expensive. This paper considers optical flow based object tracking as the most promising approach to visual object tracking.

Comprehensive video datasets are required for comparative studies of existing object tracking methods and development of novel methods. There are some publicly available video datasets created for object tracking methods evaluation: OTB (Wu et al, 2013), VOT (Kristan et al, 2016), KITTI Vision (Geiger et al, 2013). There are some publicly available video datasets intended for optical flow benchmarking: Middlebury (Baker et al, 2011), Sintel (Butler et al, 2012), Flying Things 3D (Mayer et al, 2016). These video datasets are synthetic, they lack real camera noise and blur distortions. The only real video dataset that includes optical flow ground truth is KITTI Vision dataset (Geiger et al, 2013). Thus development of object tracking methods for particular applications may require creation of new video datasets. Summary of the publicly available visual object tracking datasets is presented in Table 1. 
A video dataset must include ground truth for automatic evaluation of object tracking results. This ground truth information is usually specified manually by human using special video annotation software. Among special video annotation tools one can mention ViPER (Mariano et al, 2002), Ground Truth Editor (Sarvanko et al, 2013), Phylosis Label Editor (Philosys, 2018), ViTBAT (Biresaw et al, 2016) and Dark Label (DarkLabel, 2018). ViPER (Mariano et al, 2002) was designed in the University of Maryland. It requires manual annotation of an object bounding box in every video frame. Ground Truth Editor (Sarvanko et al, 2013) provides more comfortable user interface however it assumes user to annotate the whole video frame by frame. Phylosis Label Editor (Philosys, 2018) makes it possible to specify the bounding box corners in several video frames, then automatically calculates the bounding boxes for all the rest video frames using interpolation technique. Some restrictions of the software usage are implied by its commercial license. ViTBAT (Biresaw et al, 2016) is an open source analog of Phylosis Label Editor. It also uses interpolation, besides it makes it possible to label moving objects with point markers. Dark Label (DarkLabel, 2018) is a powerful proprietary tool that provides automatic tracking of the object bounding box from frame to frame and operates fairly well, thus user only needs to specify the object bounding box in the first video frame. Summary of the publicly available video annotation tools is presented in Table 2.

Table 1. Summary of public visual object tracking datasets

\begin{tabular}{l|l|l|l|}
\hline Dataset & $\begin{array}{l}\text { Optical flow } \\
\text { ground truth }\end{array}$ & Moving objects & Genesis \\
\hline OTB & - & miscellaneous & Reality \\
VOT-IR & - & miscellaneous & Reality \\
Kitti Vision & + & cars, pedestrians & Reality \\
Middlebury & + & toys, etc. & Synthetic \\
Sintel & + & human, birds & Synthetic \\
Flying Things 3D & + & miscellaneous & Synthetic \\
\hline
\end{tabular}

Optical flow tracking approach consists in object feature points detection and tracking within the video image. This approach involves several issues related to the feature point tracking. Some object feature points may be lost or move to background. Some background feature points may be picked up by the moving object. Besides the feature points may slide from the front part of the moving object to its back part while tracking. All these issues can cause inaccuracy in results of tracking algorithms. In order to deal with such issues one must prepare a video tracking dataset with optical flow measurement ground truth. There are few real video datasets that include such ground truth information, for instance KITTI Vision dataset (Geiger et al, 2013). Some problem domains can require creation of new video datasets that meet application-specific requirements. However, there are no publicly available video annotation tools for automatic object feature points markup which is critical for the optical flow ground truth specification. Indeed, ViTBAT (Biresaw et al, 2016) makes it possible to specify the object feature point position using interpolation which is not accurate enough and may require much manual adjustment efforts. Dark Label (DarkLabel, 2018) can automatically track the whole object bounding box but cannot track its distinct feature points. Besides, Dark Label is a proprietary software and cannot be easily extended by the scientific community.

Table 2. Summary of public video annotation tools

\begin{tabular}{l|l|l|l|l}
\hline $\begin{array}{l}\text { Annotation } \\
\text { tool }\end{array}$ & $\begin{array}{l}\text { Point } \\
\text { markers }\end{array}$ & Interpolation & $\begin{array}{l}\text { Automatic } \\
\text { tracking }\end{array}$ & License \\
\hline ViPER & - & - & - & Open \\
Ground Truth Editor & - & - & - & Open \\
Phylosis Label Editor & + & + & - & Commercial \\
ViTBAT & + & - & - & Open \\
Dark Label & - & + & + & Proprietary \\
\hline Our Tool & + & + & + & Open \\
\hline
\end{tabular}

In this work we create a new video annotation tool for semi-automatic annotation of the object bounding boxes and its feature points position within the video thus providing ground truth information for the optical flow evaluation on real videos. New video annotation tool can cope with multicamera recorded videos such that user can identify and mark a distinct moving object on several camera views corresponding to the same time moment. Finally, the developed video annotation tool is open source so community can extend its functionality anyway. 


\section{VIDEO ANNOTATION TOOL OVERVIEW}

The video annotation tool was developed using OpenCV library (Bradski and Kaehler, 2016) and Microsoft .NET Framework. The Windows Forms application loads four dynamic libraries with a managed C\# code: DisplayControl.dll, ObjectTracker.dll, MarkupProvider.dll, CameraProvider.dll. The DisplayControl.dll implements a Windows Presentation Forms visual control that makes it possible to display multiple camera views simultaneously and draw a rubber band selection interactively using mouse events. The ObjectTracker.dll implements an optical flow based object tracking method for automatic bounding box calculation in a sequence of video frames. The MarkupProvider.dll manages video annotation data in memory and provides service functions for loading and storing annotation data into an XML text file. The CameraProvider.dll provides a program interface to a dynamic library OcvWrapperMfc.dll with unmanaged native $\mathrm{C}++$ code that makes it possible to open and decode video files and calculate optical flow for automatic image feature points tracking using OpenCV library functions. All dynamic link libraries of the application can be tested independently using custom testbench applications.

The source code of the application is divided into five major classes: FileManager, DisplayManager, TraceManager, MarkupManager and CameraManager. All these classes are related to one another by means of inheritance relationship. All together the classes comprise some kind of a service stack, such that any user command initiated by a button click, a mouse movement or a menu selection can be processed sequentially by all classes of the service stack. Each class of the service stack incapsulates some part of the application functionality and has links only to those visual controls of the main form it is responsible for. Such a program code organization makes it possible to hold the visual controls close to the business logic they are supported by and to simplify the source code testing and maintenance.

The FileManager class is responsible for open and save operations as well as initialization of all the rest classes of the service stack. The DisplayManager class receives data from the lower stages of the service stack and loads data into the DisplayControl visual control. The TraceManager class implements video playback, fast forward and backward, switches between trajectory edit and view modes as well as creation of new object categories, tags and trajectories. It also implements the automatic object tracking method in its private instance of the ObjectTracker class. The MarkupManager class integrates the MarkupProvider class instance into the user interface application. The CameraManager class integrates the CameraProvider class instance. The ObjectTracker, MarkupProvider and CameraProvider classes are called from corresponding service stack classes by means of documented program interfaces. The application can be easily extended with alternative implementation of these classes thus providing support of new video sources, annotation database engines and automatic object tracking methods.

Our video annotation tool has two tracking modes. In the first mode the user specifies the feature point marker position in the current video frame. The specified feature point is automatically tracked in the subsequent video frames using pyramidal Lukas-Kanade optical flow (Bouguet, 1999) implemented in OpenCV library. In the second mode the user specifies the object bounding box corners in the current video frame. The position of the bounding box in the subsequent video frames can be estimated in three ways. In the first way the bounding box for the subsequent video frame is initialized in the same position as in the previous video frame, then the user can manually adjust its corners to fit the actual object location within the video frame. In the second way the user must specify the object bounding box corners in several distinct video frames, then its positions in the intermediate video frames are calculated using interpolation. In the third way the user can specify several object feature points along with the object bounding box, then the position of the object bounding box in the subsequent video frames is automatically calculated given the positions of the object feature points tracked using optical flow in the following way.

Let $p(i, t)=\{x(i, t), y(i, t)\}$ be a $2 \mathrm{D}$ co-ordinate vector of the $i$-th object feature point in the $t$-th video frame. Let $P(t)$ be a $2 \mathrm{D}$ co-ordinate vector of a mean object feature point position in the $t$-th video frame. Let $D(\mathrm{t})$ be a object feature points standard deviation in the $t$-th video frame.

Let $C(t)$ be a 2D co-ordinate vector of the object bounding box center in the $t$-th video frame. Then 2D co-ordinate vector of the object bounding box center in the $(t+1)$-th video frame can be calculated as follows: $C(t+1)=C(t)+P(t+1)-P(t)$.

Let $S(t)=\{w(t), h(t)\}$ be a $2 \mathrm{D}$ vector of the object bounding box width and height in the $t$-th video frame. Then 2D vector of object bounding box width and height in the $(t+1)$-th video frame can be calculated as follows: $S(t+1)=S(t) * D(t+1) / D(t)$. 
The architecture of the application makes it possible to easily extend its functionality with alternative object bounding box tracking methods if necessary.

\section{CONCLUSION}

In this work we presented a brief overview of contemporary methods for automatic object detection in videos. Despite neural network methods produced a great breakthrough in recent years, optical flow based tracking methods are still promising due to their real-time performance for embedded systems. A short survey of open video datasets for visual object tracking was also presented. There are few publicly available video datasets that provide optical flow ground truth information for optical flow algorithm benchmarking and contain real life videos that include actual camera noise and blur. New video datasets should be created for testing and improvement of optical flow based tracking methods in order to meet application-specific requirements. Publicly available video annotation tools were also revised. Lack of open source video annotation tools with functions of automatic optical flow markers and object bounding box visual tracking was noticed. Thus a new video annotation tool with such functions was proposed. The proposed video annotation tool has a five layer architecture that simplifies its testing and maintenance. Video source decoding, markup database management and visual tracking program units were implemented as a separate dynamic link libraries and are called from the main application through the documented program interfaces. This approach makes it possible to add new tracking methods, video sources and markup database engines with minimal modifications of the main application source code. The source code of the new video annotation tool will become publicly available on https:/github.com/lychkovi/multicam-annotation repository when the article will be published.

\section{ACKNOWLEDGEMENT}

This research was supported by Ministry of Science and Higher Education of the Russian Federation, R\&D state project №2.5048.2017/8.9.

\section{REFERENCES}

Allili, M.S. and Ziou, D., 2006. Object contour tracking in videos by using adaptive mixture models and shape priors. Proceedings of the International Symposium on Computational Modelling of Objects Represented in Images. Fundamentals, Methods and Applications. Coimbra, Portugal, pp. 47-52.

Anishchenko, L., 2018. Machine learning in video surveillance for fall detection. Proceedings of 2018 Ural Symposium on Biomedical Engineering, Radioelectronics and Information Technology. Yekaterinburg, Russia, pp. 99-102.

Baker, S. et al, 2011. A database and evaluation methodology for optical flow. In International Journal of Computer Vision, Vol. 92, No. 1, pp. 1-31.

Biresaw, T.A. et al, 2016. Vitbat: Video tracking and behavior annotation tool. Proceedings of 13th IEEE International Conference on Advanced Video and Signal Based Surveillance. Colorado Springs, CO, USA, pp. 295-301.

Bouguet, J.Y., 1999. Pyramidal implementation of the affine lucas kanade feature tracker description of the algorithm. Technical Report, Intel Microprocessor Research Labs.

Bradski, G. and Kaehler, A., 2016. Learning OpenCV 3. Computer Vision in C++ with the OpenCV Library. O'Reilly Media.

Butler, D.J. et al, 2012. A naturalistic open source movie for optical flow evaluation. Proceedings of European Conference on Computer Vision, Florence, Italy, pp. 611-625.

DarkLabel 1.3. Image Labeling and Annotation Tool. https://darkpgmr.tistory.com/16

Deviatkov, V.V. and Lychkov, I.I., 2017. Recognition of Dynamical Situations on the Basis of Fuzzy Finite State Machines. Proceedings of IADIS International Conferences Computer Graphics, Visualization, Computer Vision and Image Processing 2017 and Big Data Analytics, Data Mining and Computational Intelligence 2017. Lisbon, Portugal, pp. 103-109. 
International Conferences Interfaces and Human Computer Interaction 2019; Game and Entertainment Technologies 2019; and Computer Graphics, Visualization, Computer Vision and Image Processing 2019

Du, B. et al, 2018. Object Tracking in Satellite Videos Based on a Multi-Frame Optical Flow Tracker. arXiv preprint arXiv:1804.09323.

Geiger, A. et al, 2013. Vision meets robotics: The KITTI dataset. In The International Journal of Robotics Research, Vol. 32, No. 11, pp. 1231-1237.

Kristan, M. et al, 2016. A novel performance evaluation methodology for single-target trackers. In IEEE transactions on pattern analysis and machine intelligence, Vol. 38, No. 11, pp. 2137-2155.

Lychkov, I.I. et al, 2018. Tracking of Moving Objects With Regeneration of Object Feature Points. Proceedings of 2018 Global Smart Industry Conference. Chelyabinsk, Russia, pp. 1-6.

Mariano, V.Y. et al, 2002. Performance evaluation of object detection algorithms. Proceedings of IEEE International Conference on Pattern Recognition. Quebec City, Quebec, Canada, pp. 965-969.

Mayer, N. et al, 2016. A large dataset to train convolutional networks for disparity, optical flow and scene flow estimation. Proceedings of the IEEE Conference on Computer Vision and Pattern Recognition. Las Vegas, NV, USA, pp. 4040-4048

Morozov, A.A. et al, 2015. Development of concurrent object-oriented logic programming platform for the intelligent monitoring of anomalous human activities In Biomedical Engineering Systems and Technologies. Communications in Computer and Information Science, Vol. 511, pp. 82-97.

Philosys Label Editor. https://www.philosys.de/en/products/label-editor

Price, E. et al, 2018. Deep Neural Network-based Cooperative Visual Tracking through Multiple Micro Aerial Vehicles. arXiv preprint arXiv:1802.01346.

Sarvanko, J. et al, 2013. Consumer video dataset with marked head trajectories. Proceedings of the 4th ACM Multimedia Systems Conference. Oslo, Norway, pp. 84-89.

Taranyan, A.R. et al, 2018. Selective Covariance-based Human Localization, Classification and Tracking in Video Streams from Multiple Cameras. Proceedings of the 9th International Conference on Bioinformatics Models, Methods and Algorithms; Part of the 11th International Joint Conference on Biomedical Engineering Systems and Technologies. Funchal, Portugal, Vol. 3, pp. 81-88.

Wu, J. et al, 2018. Study of multiple moving targets' detection in fisheye video based on the moving blob model. In Multimedia Tools and Applications, pp. 1-20.

Wu, Y. et al, 2013. Online object tracking: A benchmark. Proceedings of the IEEE conference on computer vision and pattern recognition. Portland, Oregon, USA, pp. 2411-2418.

Yu, Y. et al, 2018. HeadFusion: 360 ${ }^{\circ}$ Head Pose tracking combining 3D Morphable Model and 3D Reconstruction. In IEEE Transactions on Pattern Analysis and Machine Intelligence. Vol. 40, No. 11, pp. 2653-2667. 